\title{
5D Model Theory for the Creating of Life Forms
}

\author{
K. W. Wong', Peter C. W. Fung'2, W. K. Chow ${ }^{3}$ \\ ${ }^{1}$ Department of Physics and Astronomy, University of Kansas, Lawrence, USA \\ ${ }^{2}$ Department of Physics, Department of Medicine, and Centre on Behavioral Health, University of Hong Kong, Hong Kong, China \\ ${ }^{3}$ Department of Building Services Engineering, The Hong Kong Polytechnic University, Hong Kong, China \\ Email: kww88ng@gmail.com
}

How to cite this paper: Wong, K.W., Fung, P.C.W. and Chow, W.K. (2019) 5D Model Theory for the Creating of Life Forms. Journal of Modern Physics, 10, 1548-1565. https://doi.org/10.4236/jmp.2019.1013103

Received: October 23, 2019

Accepted: November 17, 2019

Published: November 20, 2019

Copyright ( 2019 by author(s) and Scientific Research Publishing Inc. This work is licensed under the Creative Commons Attribution International License (CC BY 4.0).

http://creativecommons.org/licenses/by/4.0/ (c) (i) Open Access

\begin{abstract}
Based on the Fermat's Last Theorem and the Po, P1 projections from the 4th space coordinate to the time variable for Po and to the remaining $3 \mathrm{D}$ space variables for $\mathrm{P} 1$, the carbon 12 nucleus is shown explicitly as given by the hard-sphere dense packing model that also satisfies the Gell-Mann standard model. It is through these that $\mathrm{C} 12$ is a vital element in all biomaterials, and all proteins as well as the Nitrogenous bases in DNAs, are of hexagon geometric structures. Furthermore, the unique presence of a $3 \mathrm{D} \times 1 \mathrm{D}$ space void within the $\mathrm{C} 12$ nucleus provides for the monopole Boson field tunneling to occur, giving rise to the enormous variety spectra in the DNA of life forms. In addition, on the surface of the bio cells, the carbon valence band $\mathrm{p}$ electron excitation into the empty conduction band separated by a bandgap G, can result in HTC Excitonic induced superconductivity binding gaps from the Excitonic spectra, which match part of those of the DNA and thus produce the self-grow mechanism of numerous different cells in a life form.
\end{abstract}

\section{Keywords}

5D Projection, Nitrogenous Bases, DNA Spectra, Cell Growth, Genome, Protein Effect, Excitonic Superconductivity in Cells, Fractal Hexagon Carbon Representation

\section{Introduction}

We had forwarded a suggestion that "Life" can be modeled by the topological mappings from the 5D homogeneous manifold [1]. It was however only a hypothesis as in that reference neither the Perelman mappings [2] and the explicit Maxwell monopole potentials [3] were derived and incorporated into the 5D theory until recently [4]. The mappings of the homogeneous 5D manifold onto the $4 \mathrm{D}$ Lorentz manifold together with the imposing of the uncertainty principle with gauge invariance not only produce the Gell-Mann standard model because of the $4 \mathrm{D}$ space reduction projections giving rise to the Semi-simple Compact 
Lie groups, $\mathrm{SU}(3)$ and $\mathrm{SU}(2)$ [5] giving the weak lepton model [6]. Furthermore, it also was successful in producing the models for the creation of galaxies with an extremely hot circular core, that would result in the presence of two gamma ray bubbles on each side of the core, while within the galactic plane there are the stars and planets together with their properties of self-rotation and the present of dipolar magnetic field [7] [8]. In these processes charges and masses are created by splitting the energy value of the Diagonal Long Range Order monopole Bosons along the Fermat's radial, which are originally in the Bose-Einstein condensed state, and hence giving rise to the concept of Temperature via the introduction of the grand canonical ensemble, yet retaining the Lie group symmetry as a fractal hexagon geometry of the mass structures, which are created by the space dimension reduction projections. It is therefore obvious that there remains the fractal representation on the extended low energy range or longer wavelength domain of the monopoles not responsible for the initial conversion into integer charges and masses should also exist for the 5D manifold monopoles. But since such Bose states must still be projected into geometrical symmetries that are technically the same as that given by the Lie groups. A simple illustration is the hard-sphere dense packing model for the carbon 12 nucleus [9]. Therefore, we see after applying the gauge constrain on the protons and neutrons in the nucleus that the topological symmetry is the formation of 3 hexagons and 6 equilateral triangles [1]. Hence in order that the projection action can be applied also to the very long wavelength region of the monopoles, a breaking of the 3D spherical space symmetry given for the carbon nucleus into $2 \mathrm{D} \times 1 \mathrm{D}$ must be imposed. It is this further space symmetry breaking that leads us to the basic formation of the $2 \mathrm{D}$ biological Nitrogenous bases, that is realized in a correspondingly lower temperature domain from the canonical ensemble. However, due to our need to maintain the gauge invariance on the DLRO monopole Boson by completing it into a closed loop, such 2D hexagon-shaped Nitrogenous bases must be linked along the $1 \mathrm{D}$ via quantum tunneling. This results in the creation of RNAs and with the implications of an endpoint reflection into a parallel RNA and therefore completing a DNA [1], the most essential component to the building of "Life". The diversity of such DNAs formed is infinite, as the thermal parameters are varied, not to mention the infinite possible variations of the gap magnitudes between the different base layers, hence the infinite diversity of life forms. It is this most complex geometrical projected results, which we proposed as responsible for "Life" that we like to present in the current paper as the physics of "Life". In the present paper, we shall utilize these mathematical results and separate our discussion into the following separate sections linking DNAs to genome and the creation of proteins and cells, etc., and thus life forms including us humans.

\section{The Mapping of the Magnetic Monopole Field within an Enclosing Time Frozen Matter Poincare Sphere}

The Maxwell monopole fields are the quantum field result of the 5D homoge- 
neous quadratic space-time operator. It is a Diagonal Long Range Order DLRO product of two opposite " $e$ " charge massless spinors, that is along the Fermat's amplitude " $r$ " [10]. Thus its magnitude is given by "2ec". Since it is non-dependent on the eigenenergy value of the monopole Boson it must be represented in the grand canonical ensemble and thereby in the Bose-Einstein condensed state as we mentioned in the introduction. Such a state implies that the magnetic monopoles give raise to a vacuum in $4 \mathrm{D}$ space-time that is filled with this zero net charge Boson field, a quantum picture that is consistent with the Higg's vacuum [11]. We refer our readers to ref. [11] for details. Hence, should the 5D manifold be time frozen, the net 0 charge magnetic monopole 2ec along " $r$ " must be mapped onto the inner surface of the spherical void instead of being broken into 2 separate 0 and $2 \mathrm{e}$ Boson fields circulating within the $3 \mathrm{D}$ spherical void core through Po, the space to time projection that resulted in $\mathrm{SU}(2)$ for the -e leptons and the $\mathrm{SU}(3)$ via the space to space conformal projection P1, as discussed in detail in ref. [1], giving us the Gell-Mann standard model, which are resulted from the monopole energy, and thereby resulting in the rotation angular momentum state $<2 \mathrm{hvt}(0)>$ along $\mathrm{z}$, where $<>$ represents the canonical ensemble averaged, and a separate magnetic dipole $\mathrm{B}_{\mathrm{z}}$, as proportion to M.r $=\left\langle 2 \mathrm{ec}^{2} . \mathrm{t}(0)\right\rangle$. Both such results were discussed at length by us as illustration on the self rotation and dipolar magnetic fields in stars and planets [7] [8]. We again refer our readers to these two earlier publications. What we need to emphasize is matter created via Po and P1 are from monopole energies exceeding the rest mass energy of the electron and the bare quarks by the choosing of a different chemical potential value $\mathrm{m}(\mathrm{e})$ the electron rest mass for the separated negative e charge, and $\mathrm{m}(\mathrm{q})$, the quark rest mass for that of the positive e charge. The detail obtained through force balancing due to the Perelman mappings and the masses from Po and P1 are also recently published [12]. Again we refer our readers to that paper. However, the lowest quantum Poincare sphere resulted from P1, gives us the carbon 12 nucleus [13] was not carefully analyzed. In fact it was suggested in ref. [1] by making sure that the 6 protons and 6 neutrons on the C12 nucleus shell form 3 hexagons and 6 equilateral triangles, with each point triple degenerates, hence making them in the lowest Standard model that of either a proton or a neutron and makes the nucleus $\mathrm{C} 12$ the lowest symmetric Poincare sphere, with a spherical time frozen void core [Figure 1], absence of the monopole field. However within the low energy frequency region this DLRO field must remain intact, but it remains in DLRO when time is frozen, unless it can quantum tunnel from $\mathrm{C}$ to $\mathrm{C}$ and completes a closed loop, as required by gauge invariance. It is this realizable gauge state that introduces the fractal representation with the replacement of the proton and neutron in the $\mathrm{C} 12$ nucleus with a $\mathrm{C} 12$ and a Nitrogen atom sphere without a void core, such that we may generate 3 pairs of cancelling + and -Bz' along " $\mathrm{r}$ ". With 60 degree in between each $z^{\prime}(j)$. Because of charge equipotential Theorem, such quantum Bz' states will always cancel each other under perfect symmetry, 


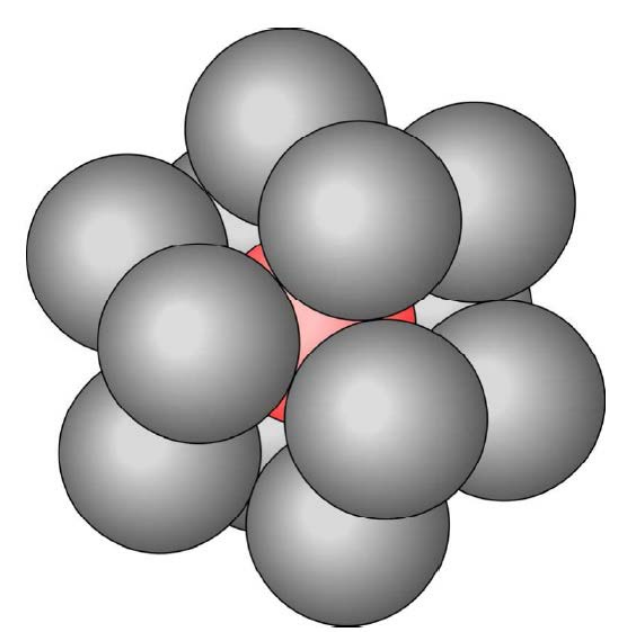

Figure 1. C12 hardsphere dense packing model (Wong et al. 2014, ref. [1]).

unless we can divide them and map the 3 hexagons produced by $\mathrm{P} 1$ onto a flat $2 \mathrm{D}$ plane. It is this topological surgery that creates 3 totally independent Nitrogenous bases, with 3 neighboring C's, and 2 N's sandwich a C [see Figure 2]. Because the 4 th $\mathrm{C}$ between the $2 \mathrm{Ns}$ only serves to create a bone structure linking stacks of such Nitrogenous bases, forming RNA structure, it cannot be considered as also a fractal representation of the proton by $\mathrm{C} 12$. Hence by recognizing the M DLRO state must remain perpendicular to the hexagon, this monopole Boson field must tunnel from one layer of the Nitrogenous base to the next, provided by wave-lengths exceeding the space gap between the two neighboring layers, which could also contain non-Nitrogenous $C$ layers, so that at the ends it must be able to be reflected onto a parallel RNA stack, thus completing a mathematically required closed loop and thus preserves the gauge invariance. For the two RNAs within the DNA end points reflection an extra reflective boundary condition hence a 4th Nitrogenous base representing the fractal of Po mapping must be introduced to make the basic set in all DNAs, 4 bases instead of 3 as depicted by fractal geometry. With such rather long wave-length spectra, because of the large number of non repeated choices $=8$ ! Of stacking of the parity pair Nitrogenous bases layers in the DNA, the equivalent temperature energy is then in the so call life suitable temperature range. In order that the stacking can complete a close loop by requiring that the two ends of the double RNA, that makes up the DNA be always those of the Thymine base, the number of non repeat stacking is given by the 4 bases with their mirror representation, as they are not necessarily parity invariant. While the gene frequency is dictated by a closed loop of the M, the DLRO Boson that quantum tunnels from the 3 adjoining C's in a base to the next $3 \mathrm{C}$ 's in the next nearest neighbors bases layers. Hence the genome number must be given by $3 \times 8 ! \times 2 / 7=34,560$. The number 3 comes from the 3 adjoining C's within each base. Since each quantum closed loop of $M$ must pass through all 8 bases states, the number of different possible path or 


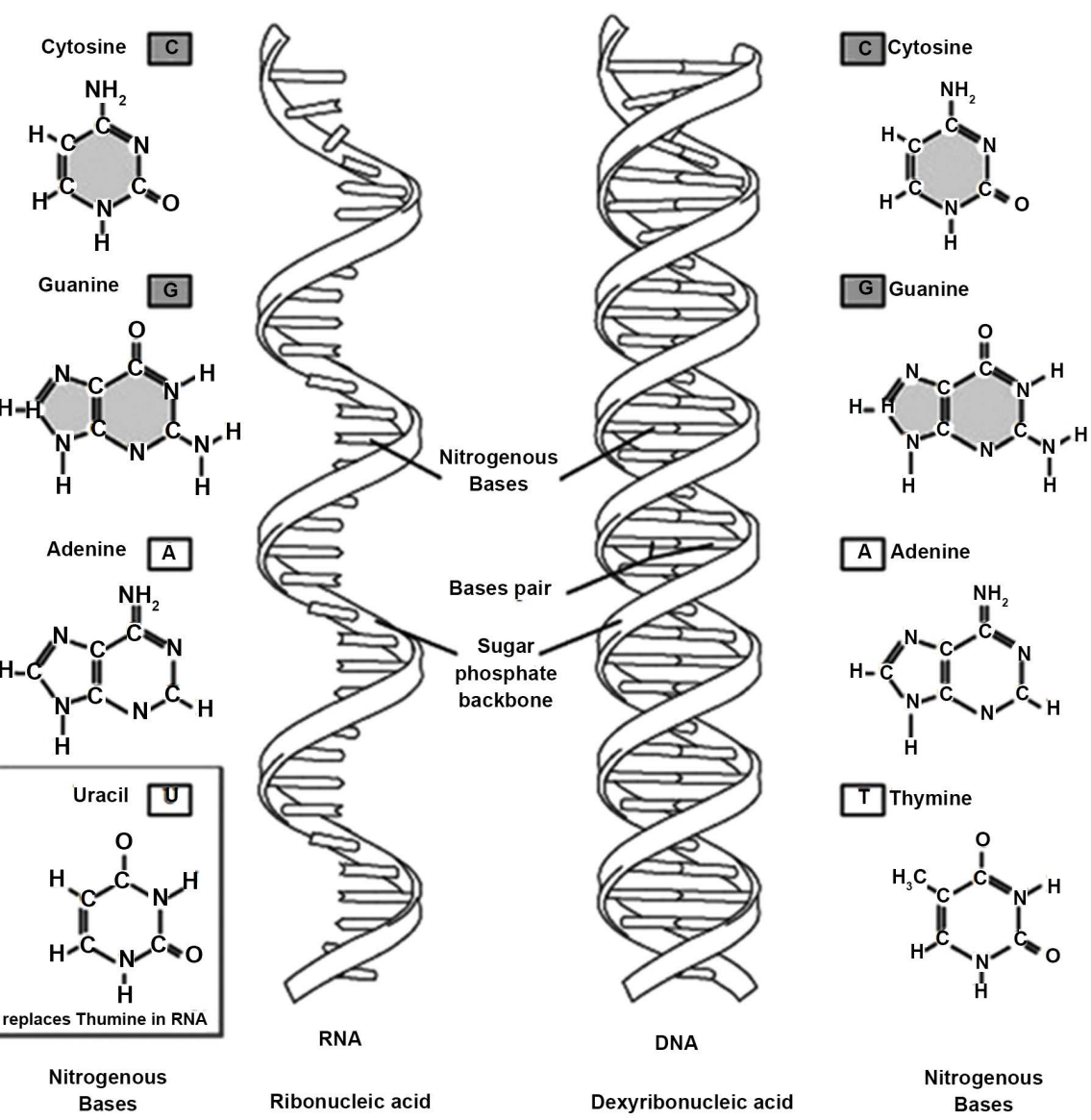

Figure 2. Images of DNA and RNA [Wong et al. 2014].

dering is 8 ! However the 2 end cap Thymine bases are parity invariant, and must be separated by 6 non Thymine bases in between within the closed loop, thus the number of distinct choices is multiplied by $2 / 7$. The 2 comes from the two Thymine bases within. The $1 / 7$ is due to the fact that once one Thymine cap is chosen then the remaining Thymine must be fixed and hence is only $1 / 7$ coming from the 7 bases locations left to choose. It is interesting to observe that this genome number appears to be universal to life forms, it's monopole $\mathrm{M}$ eigen-frequencies depend on the loop circumference, which must depend on the base to base separation gaps, as well as the DNA twisting, and thereby depend on the physical length of the DNA, that is vital to the creation as well as serves as a signature of an individual life form. Hence comparing the DNA spectra is then applicable as a verification of the ancestry of a life, because the quantum frequency spectra on the DNA loop is quite unique, with infinite possible separating gaps variations, hence no two individuals are $100 \%$ exactly identical. The key we emphasize is the quantum frequency spectra range is dictated by the stacking geometry, which not only included the multi-non-Nitrogenous in-between layers. Thus as averaged over a thermal canonical ensemble has a temperature $\mathrm{T}$ spread. It is such a $\mathrm{T}$ spread that dictates whether the DNA generated life can exist in its thermal environment. For example, sea lives due to water freezing at 0 
C. would have a narrower T distribution for its DNA then human that can survive a much wider temperature range. The wider the $\mathrm{T}$ distribution for the DNA spectra, the more complex of a life form it tends to produce.

Only roughly 30,000 genome is found in human.

\section{The Role Played by the DNA Spectra and the Natural Growth of Biological Cells}

All biological tissues contain proteins. All proteins are basically $2 \mathrm{D}$ structures of molecular hexagons containing carbons. It according to $5 \mathrm{D}$ theory are the result of Perelmann entropy mapping, combined with the chemical binding of other elements within the thermal environment, similar to the formation of galaxies, except it has no center void core, due to the dimensions of the macro-hexagons formed, hence it also does not contain self rotation and a magnetic field. Since they are fundamentally $2 \mathrm{D}$, they are very flexible and hence the elements within do not form a rigid point group symmetry. That is the electron orbitals within these protein structures do not result in a band structure. The forms of proteins are thereby purely dependent on the availability of elements within its thermal environment. Because of the fractal picture as we mentioned earlier derived from the P1 mapping of homogeneous 5D manifold, which gives us the C12 nucleus, and the basic hexagon structure, all tissues are formed in strings as can be visible from the topology of the next order Poincare sphere symmetry; namely the Bucky ball, where the hexagons are linked with isolated pentagons in between [see Figure 3]. Making biological tissues into string structure fibers. Like crystals it can be broken by temperature changes. Yet these proteins are vital to the formation of cells. Cells on the other hand are basically spherical, with dangling bounds attached to the surface, and always contain DNAs within it. Because of the spherical nature, the electron orbitals on the surface of the cell, necessarily obey periodicity and thereby form a band structure. When proteins are rapped around a DNA, the spectra of the DNA affects the transition of the cell's surface electronic states into an ODLRO, such that its binding energy matches precisely of the eigenvalues in the DNA's DLRO gauge invariant monopole Boson state. The details of this mechanism will be discussed in the next section. Since there are a large number of DNA spectra eigenvalues, there are correspondingly a large number of different cells possible. The introduction of an abnormal protein into the bio environment, can have a detrimental effect on the life itself, as it creates abnormal cells, or inhibits the normal cell formation. Hence a normal protein belonging to a specific life form, like a plant that is absent in animals can be toxic, which includes us human. On the other hand the reverse are beneficial to the animal when consumed. In fact it is the principle behind the benefits in herbal medicines.

\section{The Key Role Play by Carbon 12 in Bio Cells}

There are numerous studies and publications on many different areas in cell biology. Its functions, compositions and reproduction: Including genome and 


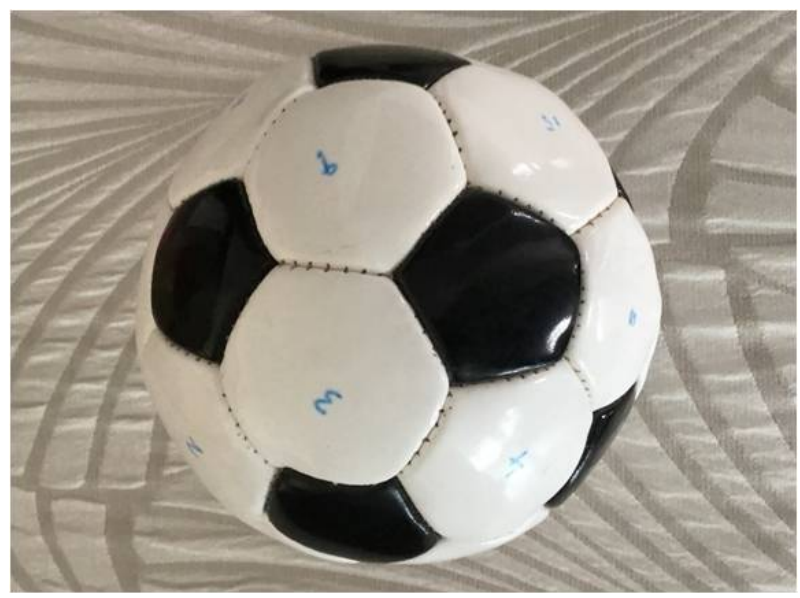

Figure 3. The 20 hexagons are in white, while the 12 isolated pentagons are in black.

chromosomes. To ignore these progresses is a huge mistake. However, there has never been any study on the creation of life from basic principles of space-time mapping and fundamental quantum fields. It is this note's intension to initiate such a study based on the fact that all known life forms are of carbon composition.

Carbon 12 is a very unique element based on the $5 \mathrm{D}$ theory [1]. Its nucleus has 6 protons and 6 neutrons, forming in terms of a hard sphere model [10] into 3 hexagons and 6 equilateral triangles on a shell, enclosing a spherical void core. From the 6 triangles, it is obvious they must contain either 2 protons with 1 neutron or 2 neutrons with one proton at the corners. Thereby the charge distribution cannot be uniform in alternate proton to neutron throughout. In fact if we consider the probability distribution on the shell of finding a charge it would be either $2 / 3$ or $1 / 3$. These fractions are actually in agreement with the P1 space to space projection model from $5 \mathrm{D}$ homogeneous space-time with uncertainty principle, where only e and -e opposite momentum pairs of massless spinors along the Fermat's amplitude can exist, such that the $+\mathrm{e}$ of the massless spinor is broken into fractional charges and gain a mass becoming quarks in the 4D Lorentz space time $\mathrm{L}$, so that the $5 \mathrm{D}$ is mapped into $\mathrm{SU}(3) \times \mathrm{L} \times 1 \mathrm{D}$ [6]. From the Fermat's Last Theorem, the homogeneous space-time $\mathrm{N}$ dimensional manifold are expressed as the amplitude $r$, with angle $0<\varphi<2 \pi$ for $2 \mathrm{D}$ space, $0<\theta<\pi, 0$ $<\varphi<2 \pi$ for 3D space, and $0<\theta<\pi, 0<\varphi<2 \pi, 0<\varepsilon<4 \pi$ for $4 \mathrm{D}$ space. Thus under the $\mathrm{P} 1$ projection from $4 \mathrm{D}$ space to $2 \mathrm{D}$ space, in terms of hard spheres, the 3 hexagons due to $\varepsilon$ and 6 equilateral triangles geometry resulted on the spherical surface. But because of the equipotential charge distribution Theorem, the superposition of the 2 equilateral triangle charge representations choices must be imposed, such that the +e charge is broken into the set of SU(3) generators: $+2 / 3$ e, $+2 / 3$ e and $-1 / 3$ e, which is equivalent to the realization of gauge invariance preserving unit $+\mathrm{e}$. And at the vertex points of each of the 3 hexagons representing the $\varepsilon$ phase, which has a total of $4 \pi$ internal net angle, thus it is a 
geometric realization that represents the 4 th space dimension as according to the Fermat's amplitude and phase angle representation.

While $\mathrm{C} 12$ nucleus is composed of 18 up and down quarks each, divided into 3 hexagons, and making every vertex point containing 3 quarks that satisfy gauge invariance, such that the $\mathrm{C} 12$ nucleus is actually a quantum quark soup. Thus on the C12 shell, we have exactly 6 protons and 6 neutrons in agreement to the nuclear shell model. So that leaving only within the $\mathrm{C} 12$ void, the radial outward net 0 charge and massless Bose magnetic monopole fields $\mathrm{M}=+,-2 \mathrm{ec}$ can remain to exist [3]. While the projection Po from the 4 th space coordinate onto time must produce the $6 \mathrm{SU}(2)$-e electrons so as to maintain charge neutrality of the $5 \mathrm{D}$ manifold from which $\mathrm{C} 12$ is created by space dimension reduction projection. Hence in the atom $\mathrm{C} 12$ gives the 6 electrons revolving around the nucleus. It should be mentioned that if a spherical $3 \mathrm{D} \times 1 \mathrm{D}$ time frozen void exists within the earth crest, and if fractal representation is valid, not considering charge, a very unexpected rock formation of hexagonal shaped columns composing of mainly 2 elements within the mass of granite rock due to P1 and Po due to 4th space dimension reduction mapping [4] could be formed even from volcanic eruptions after the molten lava cools. This was of course found throughout the earth's surface [see geological data in south China, HK history museum]. In yet another unrelated natural compound, is the Lithium carbonate, LiCO, a key ingredient for the Lithium battery is based on the relatively low photon energy ionization of Lithium, and it is easily recharged, because of the storage of magnetic monopole tunneling energy within the carbon on top of the carbon hexagon loop structure due to the carbonate structural composition, that can also readily converts back to the ionization photon to restore the battery charge. Such Lithium carbonate is found natural in the Brien salt field, which reviews on the surface the hexagonal structure, a fractal representation of the Sodium carbonate [see National Geographic, Feb. 2019]. Implying the topological importance of the hexagon structure due to the conformal space to space projection is valid in all energy range, and not just for the standard model of hadrons.

The Carbon element is a covalent atom, in fact the most important covalent element in nature. To understand how, let's go back to the basic Bohr atomic model. The eigen values of these 6 atomic electrons are according to Bohr's model, given by - $Z R / n^{\wedge} 2$, where $n$ is a positive integer number, $R$ is the Rydberg constant and $Z$ the $n$ level effective charge number. Thus for $n=1$, level $Z=$ 6 , and for $\mathrm{n}=2, \mathrm{Z}=4$, as it is reduced by the $2 \mathrm{n}=1$ inner s orbital level electrons shielding. Therefore for $\mathrm{C}$ with 6 electrons, its 4 outermost orbital level must be given by $\mathrm{n}=2$, with $2 \mathrm{~s}$ states and $2 \mathrm{p}$ states. Since the $\mathrm{p}$ orbitals are given by $2 j+1$, where $j=1+s$, it is $1 / 2$ full, while $s$ is filled. This means carbon's atomic outermost $\mathrm{p}$ and $\mathrm{s}$ orbital electrons can be shared with its molecular neighbors in binding provided from its valence states by either filling the remainder 2 unoccupied quantum $\mathrm{p}$ angular momentum states or by giving its $2 \mathrm{p}$ or $2 s$ to the binding neighbors as energetically allowed. This $1 / 2$ filled $p$ or empty s after binding is reflected as an example by the photo absorption ob- 
served in graphite, because of the continuous frequency spectrum allowed in a half filled VB, making graphite appears as black. Since the graphite structure is a stacking of weakly bond 2D C square lattice, which means it obeys space symmetry of $1 \mathrm{D} \times 1 \mathrm{D}$ geometry. And since $1 \mathrm{D}$ does not allow for angular eigenvalues, hence in graphite the unoccupied VB band must be the s states.

Because basically the s hole state is flat, it has very large effective mass, thus making graphite a very poor hole conductor. However, $\mathrm{K}$ inoculated graphite is a superconductor with a very low Tc temperature. This LTC superconductivity mechanism of $\mathrm{K}$ inoculated graphite has never been properly explained in the literature. It is actually easy to explain the mechanism, as that inserted $\mathrm{K}+$ ion must give away its s electron to the $\mathrm{C}$ atoms in the inoculated graphite filling a $\mathrm{s}$ hole between the layers as given in the VB band, making it $2 \mathrm{D} \times 1 \mathrm{D}$ like, thus slightly changes the hole density composition, by exchanging $s$ and creating a small fraction of $\mathrm{p}$ density in the residual VB hole density. But because this density of the $\mathrm{p}$ hole created due to inoculation is so small, the 2D like Exciton that can exist in the band gap $\mathrm{G}$ by the electric dipolar exciting from $\mathrm{VB}$ an electron into the empty $\mathrm{CB}$, caused by the VB p hole coupling is also weak, thereby according to the EEM [Excitonic Enhancement Mechanism] mechanism, [14] produces [the EEM theory was introduced for the ceramic HTC materials, and has been extensively investigated both theoretically and experimentally [15]-[21] An example list of such works is given in our references. Readers in particular from the bio sciences, interested are advised to read them] a quantitatively low Tc. Hence such a $\mathrm{K}$ inoculate dgraphite superconductor, despite the low Tc value, actually is due to the same EEM mechanism that produced the ceramic HTC, and not that of phonon induced BCS mechanism in superconducting metals [22].

In fact since in general molecular bio structures of $\mathrm{C}$, the $\mathrm{C} p$ orbital electrons with lattice geometry consisted of hexagons it can equally share either as a negative valency state in molecular binding, or a positive valency and accept an extra electron to fill its $\mathrm{p}$ angular momentum shell irrespective to the rest of the nearest neighbors geometry. In such more general molecular bio chemical rigid structure, the C's in the structure would naturally produce a partially filled $\mathrm{p}$ VB structure, when the bio carbon forms a solid structure with translational unit cell symmetry. But unlike that in graphite, because of the VB half filled p states will not have large effective masses. In fact because by changing the 2D C net from square to hexagon, changes the number of nearest neighbors from 4 in square net to 3 in hexagon net, leaving the VB with at least one $\mathrm{p}$ hole, which can have relatively low effective mass. All proteins that make up bio cells have natural hexagon $\mathrm{C}$ structures, except free proteins are not rigid.

Therefore in free proteins, the electron periodic boundary condition needed for band theory is not met, and hence in free proteins we cannot produce defined band structures. On the other hand, all bio cells are basically 3D spheres and contain carbons from the proteins coating in its molecular surface structure, which can be made rigid and enclosing a DNA within it. And in a fractal way, by 
taking $\mathrm{C}$ as replacing the proton in the $\mathrm{C} 12$ structural form, because of the $\mathrm{M}$ state within DNA core, and the $\mathrm{P} 1$ that broke the $3 \mathrm{D} \times 1 \mathrm{D}$ space symmetry, producing the hexagon structure shell for the cell, giving us the band structure for such cells, which are in a macro scale in the geometric shape of a sphere except also normally with different $1 \mathrm{D}$ like spike attachments. The effect of such attachments does not break the spherical surface periodicity on the electronic states from the $\mathrm{C} 12$ atoms, but like $\mathrm{K}$ inoculated graphite it would modify the VB $\mathrm{p}$ wave density and give a perturbative correction to the exciton spectrum formed within the band gap G [23]. Such minor spectrum changes are vital to the perfect matching to the DNA in the core spectra, and thus able to induce the electrons ODLRO [Off-Diagonal Long Range Order] in the specific cell, and with it the Tc value. It is such spikes that can also give the cells different functions as according to the DNA and its ability to be linked and build an organ structure. A change in Tc upward can result in inducing a cancerous growth of such a cell. The specific organ forming requires specific boundary conditions imposed not just from the DNA spectrum. Such complex boundary usually includes the chromosomes, etc. Are beyond the scope of this simple note. An example is the form of the free white blood immune defensive cell. It does not come from the VB p holes, very likely such memory comes from the semi-conductor portion on the attaching surface, similar to the LCD screen of a TV? Thus such surface semiconductor circuit memory works like a computer chip, and produces the life form's memories. It is all throughout the body of the life form, not just in the brain. The body chromosomes are examples. Although the brain cells are unique, in that its circuit accepts external programming commands, and not just play the role of the organ and shape formation. Its purpose is mainly to act as a conducting net for the stored commends in the brain provided by programmed storage key. Thereby the brain cells are generally dense packed, such that super currents can propagate on its surface similar to the granular dense pack HTC [24]. It is this physical structure that makes neuro cells rejuvenation rather rare, and its death enhanced by usage, thus increases with aging [25]. Most other bio cells, are free floating in body fluids, and it is replaced frequently, as the DNA responsible is enclosed by the specific protein that forms it. While the dead cell, breaks apart, and the protein cover is oxidized and removed from the body as $\mathrm{CO}_{2}$ and other gases emission. Because, there are few blood vessels throughout the brain, the REST protein released from death brain cells usually will accumulate and covers the brain structure, thus blocking the super current flow. It is this current blockage that leads to human neuro deceases such as Alzheimer and Parkinson. If our explanation of the cause of such deceases is valid, then we can suggest a method of control, but not a cure, if we can somehow increase the oxidation of the REST protein? Since we have no way to increasing the number of blood vessels around the brain, the only way to increase oxygen then is to enhance the blood flow. As is well known, that consuming alcohol leads to intoxication due to alcohol tends to flow to the brain. Hence, if the alcohol contains a strong oxidation agent, such as red grape wine, 
then perhaps by controlled wine drinking might keep the REST protein quantity in check? Of course such method must be tested, and success might be very limited! Thus the brain cells are susceptible to blockages due to surface coating of extra proteins, not necessarily becoming cancerous and yet leading to memory loss. Nonetheless all the cells surfaces are fundamentally spherical, and all the electronic states on its surface can be periodic as the basic chemical molecules on it always satisfy $2 \mathrm{D}$ periodic boundary, even if it is only a single unit of a cell, like for example, a Bucky ball with just certain fixed points on the surface being that of C. In short, the electron structure from them would satisfy the boundary required for band structure calculation. As such, and with the covariant character of the carbon orbitals in such spherical chemical molecules, would necessarily give rise to a partially filled $\mathrm{p}$ state $\mathrm{VB}$, together with an empty $\mathrm{CB}$, which will have from $\mathrm{n}=3$ atomic level $\mathrm{d}$ orbitals, very much like in the HTC cuprates. Since the bio cell normally will not combine with a free foreign atom, unless the free atom attachment can bring down the binding energy in excess of the band gap G between the cell's CB and VB, otherwise such a cell will not be stable when submerged in a fluid containing free ions, in bio terms, known as free radicals, when there is no positive band gap energy G, separating CB with VB of the cell band structure. Such a band structure with positive G resembles that of HTC cuprates, both band structures consisted of partially VB, with 2D like p hole conductivity in the normal phase, except in HTC the anisotropic conductivity is the result of layers of $\mathrm{CuO}$ planes stacking, while in bio-cells it is mainly due to C's on the spherical surface [23]. The molecular orbital for $\mathrm{CuO}$ in the bands gives a partial filled $\mathrm{p}$ and $\mathrm{d}$ alternating states, where $\mathrm{p}$ comes from the oxygen ion, unlike in graphite, thus with relatively small effective masses [14]. In fact the experimental result on Tc dependence on oxygen deficiency in YBCO supports our EEM theory interpretation for HTC mechanism.

Since the excitation of an electron from VB to the empty CB would form an exciton state, within the positive bandgap G, because such atomic like 2D exciton due to the cell's periodic boundary, if formed is equally periodic on the structural surface which as required for formation of band structure, it can actually couple to any moving phole by the electrical dipole induced by the Photon transition excitation of the orbital states caused by the hole charge motion. It is this electrical dipole coupling between the periodic exciton and the mobile holes that will produce EEM and thereby creating the superconducting phase transition for temperature below its superconductivity critical Tc [15]. Since Tcdepends on the Excitonic dipolar coupling, it therefore is indirectly dependent on the exciton's quantum orbital states. That is the set of reduced charge quantum orbital excitation transitions. Hence when such photonics spectrum photons are made available from the enclosed DNA, the cell would naturally form when the protein molecules that it is made up of are available within the thermal bath, so that the bio structure can minimize its carrier ground state binding gap energy matching part of the DNA spectra according to that of the enclosed DNA.

The lower the set of photo energy transitions, the easier it will be for the $p$ 
holes in the cell ODLRO condensation to occur, leading to the natural growth of the cell. In fact, the closer the exciton level is to $\mathrm{CB}$, the higher would be the specific cell's Tc [23]. Meaning, the higher Tc value as compared to the thermal bath temperature $\mathrm{T}$, and the more stable it is for the cell. None the less the EEM induced Tc has an upper limit, due to the optimum half filled VB p state, and its effective lowest possible mass, as due to the unit cell's size. Hence all life forms can only exist within this limited upper Tc range, normally below the boiling point of water. While the lower temperature limit is bounded by water freezing point, as water provides the fluid for moving the free proteins around the bio structure. Heat generation within a bio system, usually caused by Oxygen and other chemical reactions between the body fluid ions, such as free ions from proteins, which are $\mathrm{C}$ hexagon structures, but flexible without fixed bond lengths and can be transformed by wrapping onto a spherical structure cell, thus changes it, plus sugars which are just $\mathrm{C}$, silicate, and amino acid etc. that can make it rigid, and could also destroy the original cell structure thus breaking the $\mathrm{p}$ holes ODLRO phase, hence leading to the natural cell death. In fact, such stiffening of the shell of the specific protein is vital to the cell normal growth and death. To that water plays as a facilitator, an indispensable role in the chemistry. Chinese traditional medicines rely on identifying the specific protein associated with the Heath of the specific cells that is responsible for the specific organ operation, thus hoping by such direct supply of the needed protein can restore the organ's proper function. Although overabundance of such free radicals within the body fluids, will cause change in the normal cell regrowth cycle, and will cause health problems, like diabetes, Alzheimer and Parkinson's decease when the protein wraps over a brain cell is improbably stiffened with excessive silicate, causing memory loss and abnormal aging. However, the replenishment of the body fluid, with proteins coming from food intake is an essential component to the regrow of the shell of a cell around the DNA, and is equally also important to the body organs that must come from the build-up of many different cells via also providing the inter linkages, including the rigid silicate bone structures precisely maintained within such complex thermal cycles that operates in the bio system to create a healthy life.

Should the thermal bath temperature rise beyond the cell's superconducting Tc, due to inflammation, normal cell growth would be prohibited, and only abnormal cancerous ones survive. Hence when the body suffers from sickness, it is usually accompanied with at least a localized rise in temperature, the occurrence of inflammation there inside the body. Actually, the thermal condition and the availability of the proteins and chemicals drives the species evolution, or the minor topological mapping of cells, etc. towards its adaptation to survival. In order to apply our Tc model principle to the cause and potential cures of the body, all the exact normal cell structure and thereby its band structures must be obtained, so that the proper Excitonic spectra that can be formed within the corresponding band gap must be derived and matched to the body's DNA spectra. RNAs and DNAs of bio systems are made from 4 basic Nitrogenous bases, 
stacked and formed by back bones into rigid structures. These bases are all composed of hexagons with $3 \mathrm{C}$ carbons, with $1 \mathrm{C}$ carbon isolated from the others with an $\mathrm{N}$ nitrogen in between [see figures]. Since in our 5D model, it is the Po and P1 projection operations that lead to converting the massless charged spinors into $\mathrm{SU}(2)$ gauge conserved leptons and $\mathrm{SU}(3)$ with fractional charged quarks that create matter, thereby through the Perelman-entropy mapping giving us the Nitrogenous base structures, are divided into $3+1$ distinct attachments as shown in the figures. The 1 represents the end base cap, responsible for reflecting the $\mathrm{M}$ field from one RNA branch into the parallel one, while the 3 together with its opposite sides represent the $6 \mathrm{SU}(3)$ generators, since each $\mathrm{C}$ contain a time frozen $3 \mathrm{D} \times 1 \mathrm{D}$ void, where the magnetic monopole Boson State $\mathrm{M}=2 \mathrm{ec}$ can exist if it can tunnel from $\mathrm{C}$ to $\mathrm{C}$ and completes a closed loop, conserving gauge invariance by the stacking of two parallel chains of RNAs, with an end cap base to satisfy the formation of a DNA.

\section{Summary on the 5D Projection Processes}

Because the creation of 'Life' is the most challenging question, yet we believe it can be explained based on the assumption that the universe started with the creation of a single homogeneous 5D space-time manifold, which satisfies the Fermat's Last Theorem [10], together with the uncertainty principle on measurement, thus leading to the Big Bang theory, it is best to give a summary to the steps that leads to all creations: 1) The quantum field solutions of the 5D homogeneous manifold are those of the Electro-magnetic 4 vector potentials, plus the extra magnetic monopole potential as suggested by Maxwell. This Maxwell monopole magnitude is a Bose field of 2ec from the product of e and -e massless spinors, where e is the coupling between the Electro-magnetic potentials and the massless spinor, thus must obey the gauge transformation. 2) Since the Fermat's sum is only valid for positively increasing time, these vector potentials are not time reversal invariant. And it also means, parity symmetry need not be rigorously obeyed. 3) The magnetic monopole Boson is energy independent, and is of Diagonal Long Range Order, therefore, it must be in the Bose-Einstein condensed state. But to become that it must be in a Grand Canonical Ensemble, thus making up a chemical potential value in exact cancellation to the Boson's energy. 4) The fixing and choosing of a discrete set of chemical potentials break the Boson ground state, similar to the Higg's vacuum theory [11] [26] by breaking the continuous chemical potential of the Grand Canonical Ensemble into a discrete set. 5) The creation of mass matter, is then identified with this chemical potential set, and is an irreversible process, equivalent to the space reduction projection, and the Perelman mappings. Hence one ties the irreversible mapping for the creation of mass to the creating of Lorentz $4 \mathrm{D}$ manifolds out of the original single homogeneous $5 \mathrm{D}$ manifold. As well as identifying the breaking of the net charge 0 , monopole Boson into separate $+e$ and -e massive spinors [1]. Detail on the symmetry breaking had been discussed in earlier publications and will not be 
repeated here. Nonetheless it clearly divided the energy range of the monopole Bosons, into a range when its energy is greater than the rest mass of the electron, the lightest massive particle due to the projection to the lower energies. 6) Due to the rest mass differences between the $+\mathrm{e}$ and $-\mathrm{e}$ massive spinors generated by the Boson ground state excitation, the resulting atomic elements, which composed of protons, neutrons and electrons no longer maintain charge and parity symmetry [27]. 7) Since the breaking of the continuous energy range for the monopoles due to uncertainty principle also implies a breaking of the continuous $4 \mathrm{D}$ homogeneous space, thus would result in the separate formation of doughnut disc like Lorentz manifolds, which in turn divided the universe into disjoint Lorentz manifolds, with multiple energy source composed of the distribution of monopole Bosons, from which both $+\mathrm{e}$ and $-\mathrm{e}$ masses can be created. Below the rest electron masse region of the Monopole Bosons, it is obviously of longer wave-lengths. Thus under the Perelman entropy mapping [3], Poincare spheres can be obtained, with a resulting time frozen void core, and the forming of masses into three hexagons shape due to SU(3). While the void core dimension restricts the existence of the monopole Boson field. 8) The breaking of the spherical masses on the shell further splits these 3 symmetric hexagons into $32 \mathrm{D}$ separate hexagons, separated along the vertical axis of the 2D plane. 9) In life forms, we are interested in the monopole state presence due to the void of carbon 12 nucleus, as all life forms are basically carbon compounds. Since the carbon 12 void is of nucleus dimension, the monopole Boson would have to have a short wave-length and does not exist normally, unless it can quantum tunnel from one $\mathrm{C}$ in one hexagon onto another $\mathrm{C}$ in the adjacent hexagon layer, thus creating the RNA, except with fixed end points, as represented by the Nitrogenous bases set: Cytosine, which comes from the fractal representation of the hexagon on the C12 nucleus surface [Figure 2], while Guanine, Adenine are from those on the Bucky Ball surface [Figure 3], due to the 2:1 ratio between numbers of pentagons in the Bucky ball to the equilateral triangles in C12, and the 1D end cap Uracil, such that the monopole Boson is a standing wave [Figure 1]. Hence this Boson state violates gauge invariance. 10. In order to retain gauge invariance for the Boson wave, we need to change the end cap hexagon into a reflecting cap, Thymine, such that the Boson state from one RNA reflects onto a parallel RNA, and becomes a closed loop forming a DNA 11. The non-repeat stacking of the bases then gives us the genome, with its unique spectra for each life form. 12 . This unique quantized gauge solution set of the genome spectra for the monopole Bosons allows the computation on the thermal temperature $\mathrm{T}$ by using $1 \mathrm{D}$ bose distribution averaging within the life form's body, [note that 0 frequency state is always excluded, such the genome spectra does not B. E. condense] and in order that cells can be generated by the EEM mechanism for the partially VB $\mathrm{p}$ holes from the carbons in the protein shell the superconducting critical Tc must exceed $\mathrm{T}$. Yet not too far as to affect the normal cell replacement cycle caused by oxidation, and other chemical reactions. This balancing of $\mathrm{T}$ criteria in 
facts dictates the well being of the life.

\section{Conclusions}

Under the homogeneous 5D space-time quantum theory, the creation of matter from the orthogonal 4th space Bose-Einstein condensed magnetic monopole Boson fields, in the Grand Canonical Ensemble, due to space dimension reduction into Matter filled Lorentz 4D, with 5D voids, can be divided into different energy regions, and with high energies above electron rest mass, splitting the equal energy values of + and -e massless spinors with the different chemical potential values according to the electron rest mass and the quark rest mass for the different charges in the monopole field amplitude in the Grand Canonical ensembles leading to the realization of the Gell-Mann Quark model as well as the electro-weak lepton model, which we had previously published. It also can be compared to the breaking of the Higg's vacuum. Thereby, there remains the energy region below the electron rest mass, which can be shown to further split into $2 \mathrm{D}$ space matter geometry of hexagon structure when the remaining $3 \mathrm{D}$ homogeneous space is broken into $2 \mathrm{D} \times 1 \mathrm{D}$ representation. This $2 \mathrm{D}$ space includes that of a spherical surface, and that of a cylindrical cross section representation which would result the lowest symmetry geometries, that of the nucleus C12 shell, that consisted of protons and neutrons in 3 hexagons and 6 equilateral triangles and for the cylindrical geometry 4 basic Nitrogenous bases, formed by carbons and nitrogens also in hexagon form as the result of fractal where the nucleons on $\mathrm{C} 12$ shell are replaced by the carbon and nitrogen atoms that are spaced along the 1D, thus creating both RNAs and DNAs. It is through these results and gauge invariance genome and chromosomes of life forms can be obtained. The distinct discrete set of such spectra coming from the Bose-Einstein condensed DLRO of the monopole Boson field would then induce the enclosing matter surface elements' $\mathrm{CB}$ electrons, or holes of unfilled VB to become superconducting ODLRO. Hence from the 5D quantum model, the Quantum Long Range Order continuity causes the DNA spectrum energy to match the ODLRO electron or hole pairing energy thus creats cell growth, and thereby "Life". In fact this space symmetry reduction is equivalent to the topological projections of Po and P1, discussed in the 5D unified field theory book [1].

As such the P1 created $M$ tunneling states made $C$ into the fractal representation as given by the 3 adjoining C's in the Nitrogenous bases of the SU(3) quark generators, while the Po was represented by the isolated C. Since Pogives raise to the $\mathrm{SU}(2)$ electron generator, that would be in the atomic orbital and responsible to the molecular binding, so this isolated $\mathrm{C}$ acts to hold the Nitrogenous bases stacking together, creating the back bone and hence must be responsible for the RNA/DNA twisting back bone required to make them rigid, and hence fixes the allowed $\mathrm{M}$ eigen spectrum. Due to the many possible Nitrogenous bases stacking order possible, together with the twisting as well as various layers to layer sepa- 
ration gaps, the eigen spectra of $\mathrm{M}$ is extremely large, enabling it to match that required to form the exciton induced ODLRO gap in the cells. Hence, using suitable RNAi technology it is possible to correct and cure all unnatural cancerous growth, and cell inflammation caused by mismatching of the DNA spectrum with the cell ODLRO gap formation. Although this is a very tedious computational problem, as there are numerous different cells, which serves to maintain the proper functioning of different organs and senses in the life forms. Nonetheless it is an achievable task with our current $5 \mathrm{G}$ computer computational power. It is to this objective end, that we hope if our 5D model theory for life is correct, can help bring about a quantifiable medical revolution method in cure for many yet incurable illnesses? Should our model be correct, this enormous work would make it the greatest technological revolution in human history. The quantitative spectra matching between DNA and the bio exciton states would identify all the life forms functioning and its senses, including the brain's memories. As a result, there are numerous patents to be filed, and will involve collaborations between cell biologists, band structure computational physicists, computer engineers, programmers and RNAi medical and bio technicians. The results would produce advances in new technology from agriculture to human health. We, as pure scientists should lead this revolution, bringing it unconditionally to the world, instead of letting selfish politicians gain control, which could lead to human annihilating world war just so they can use the technology to satisfy their ambition in gaining power for world dominance!

\section{Acknowledgements}

We sincerely thank Ms. W. L. So for her help in typing and editing this paper. Simultaneously we thank Professor Dreschhoff and Professor Jungner for insightful discussions.

\section{Conflicts of Interest}

The authors declare no conflicts of interest regarding the publication of this paper.

\section{References}

[1] Wong, K.W., Dreschhoff, G.A.M. and Jungner, H. (2014) The Five Dimension Space-Time Universe-A Creation and Grand Unified Field Theory Model. Scientific Research Publishing, Wuhan.

[2] Diklan, J. (2019) You Are Disturbing Me-I’m Picking Mushrooms! Academia.edu. Jan. 2019.

[3] Wong, K.W., Dreschhoff, G., Jungner, H., Fung, P.C.W. and Chow, W.K. (2018) The Magnetic Monopole in 5D Homogenous Space-Time. Physics Essays, 31, 493-495. https://doi.org/10.4006/0836-1398-31.4.493

[4] Wong, K.W., Fung, P.C.W. and Chow, W.K. (2019) A Quantum Representation of the Homogeneous 5D Manifold and the Perelman Mappings of 5D onto Non-Homogeneous Lorentz 4D Manifolds. Journal of Modern Physics, 10, 557-575. 
https://doi.org/10.4236/jmp.2019.105039

[5] Wong, K.W., Dreschhoff, G. and Jungner, H. (2012) On Neutron Oscillation and Predicting the $125 \mathrm{GeV}$. Two Photon Emission State from p-p Collision Based on the 5D Homogeneous Space-Time Projection Model. Journal of Modern Physics, 3, 1450-1457. https://doi.org/10.4236/jmp.2012.310179

[6] Gell-Mann, M. (1964) Nonleptonic Weak Decays and the Eightfold Way. Physical Review Letters, 12, 155-156. https://doi.org/10.1103/PhysRevLett.12.155

[7] Fung, P.C.W. and Wong, K.W. (2017) Origin of Magnetic Fields of Stellar Objects in the Universe Based on the 5D Projection Theory. Journal of Modern Physics, 8, 668-746. https://doi.org/10.4236/jmp.2017.84045

[8] Fung, P.C.W. and Wong, K.W. (2015) On the Origin of Mass and Angular Momentum of Stellar Objects in the Universe. Journal of Modern Physics, 6, 2303-2341. https://doi.org/10.4236/jmp.2015.615235

[9] Wong, K.W. (1964) Application of Nonlocal Field Operators to a System of Hard Sphere Bose Gas. Journal of Mathematical Physics, 5, 637-642. https://doi.org/10.1063/1.1704157

[10] Aczel, A.D. (1997) Fermat's Last Theorem: Unlocking the Secret of an Ancient Mathematical Problem. Penguin, London, $147 \mathrm{p}$.

[11] Higgs, P.W. (1964) Broken Symmetries and the Masses of Gauge Bosons. Physical Review Letters, 18, 305. https://doi.org/10.1103/PhysRevLett.13.508

[12] Perelman, G. (2002) The Entropy Formula for the Ricci Flow and Its Geometric Applications.

[13] Perelman, G. (2003) Ricci Flow with Surgery on Three-Manifolds.

[14] Ching, W.Y., Xu, Y.N., Zhao, G.-L., Wong, K.W. and Zandiehnadem, F. (1987) Electronic Structure and Excitonic-Enhanced Superconducting Mechanism in $\mathrm{YBa}_{2} \mathrm{Cu}_{3} \mathrm{O}_{7-\delta}$. Physical Review Letters, 59, 1333-1336. https://doi.org/10.1103/PhysRevLett.59.1333

[15] Wong, K.W. and Ching, W.Y. (2004) A Structural-Based Microscopic Theory on High-Temperature Cuprate Superconductors. Physica C, 416, 47-67. https://doi.org/10.1016/j.physc.2004.09.003

[16] Zhao, G.L., Xu, Y., Ching, W.Y. and Wong, K.W. (1987) Theoretical Calculation of Optical Properties of Y-Ba-Cu-O Superconductor. Physical Review B, 36, 7203-7206. https://doi.org/10.1103/PhysRevB.36.7203

[17] Wong, K.W. and Ching, W.Y. (1988) Off-Diagonal-Long-Range-Ordering and the Excitonic-Enhancement Model for High Tc Ceramic Oxides. Proceedings of the $3 \mathrm{rd}$ Asia Pacific Physics Conference, Vol. 1, 58-72.

[18] Wong, K.W. and Ching, W.Y. (1988) Thermodynamics of Simultaneous-Excitonic Superconductivity Condensate. Physica C, 152, 397-400. https://doi.org/10.1016/0921-4534(88)90043-3

[19] Wong, K.W. and Ching, W.Y. (1989) The Theory of Simultaneous Excitonic-Superconductivity Condensation. Physica C, 158, 1-14. https://doi.org/10.1016/0921-4534(89)90294-3

[20] Wong, K.W. and Ching, W.Y. (1989) Theory of Simultaneous Excitonic-Superconductivity Condensation II: Experimental Evidences and Stoichiometric Interpretations. Physica $C, 158,15-31$.

https://doi.org/10.1016/0921-4534(89)90295-5

[21] Wong, K.W., Fung, P.C.W., Yeung, Y.H. and Kwok, W.Y. (1992) Analysis of the Isotope Effect of High-Tc Ceramic $\mathrm{YBa}_{2-\mathrm{x}} \mathrm{La}_{\mathrm{x}} \mathrm{Cu}_{3} \mathrm{O}_{7}$ Using the Excitonic-Enhancement 
Model. Physical Review B, 45, 13017-13024.

https://doi.org/10.1103/PhysRevB.45.13017

[22] Bardeen, J., Cooper, L.N. and Schrieffer, J.R. (1957) Theory of Superconductivity. Physical Review, 108, 1175-1204. https://doi.org/10.1103/PhysRev.108.1175

[23] Wong, K.W. and Curatolo, S. (2008) EEM: The Exciton Enhancement Mechanism Theory and Experimental Evidence of Optically Enhanced Tc in High Tc Superconductors. In: Chang, O.A., Ed., Progress in Superconductivity Research, Nova Science Publishers, Inc., Hauppauge, 55-78.

[24] Fan, C.-X. (1990) Dispersive-Mode Theory for the High-Temperature rf Superconducting Quantum Interference Device. Physical Review B, 41, 2041-2045. https://doi.org/10.1103/PhysRevB.41.2041

[25] Johnson, C.Y. (2019) Excessive Brain Activity Linked to a Shorter Life. The Washington Post.

[26] Adams, T., et al. (2011) Opera Collaboration.

[27] Lee, T.D. and Yang, C.N. (1956) Question of Parity Conservation in Weak Interactions. Physical Review, 104, 254-258. https://doi.org/10.1103/PhysRev.104.254 